Available online on 25.06.2019 at http://ajprd.com
(c) 2013-19, publisher and license AJPRD, This is an Open Access article which permits unrestricted non-
commercial use, provided the original work is properly cited

Open $\overbrace{\text { Access }}$

Review Article

\title{
Use of Nanoparticles for the Treatment of Malignant Neoplasm As a Cancer Targeted Drug Delivery System
}

\section{Mohammad Khan Gayoor *, Yezdani Umama, Kumar Ayush, Sadar Mayur, Rav Shorabh, Sahu Lalit}

${ }^{1}$ Department of Pharmacy, Truba Institute of Pharmacy, Bhopal- 462038, India.

${ }^{2}$ Department of Pharmacy Practice, MRM college of Pharmacy, Hyderabad -500059, India.

${ }^{3}$ Department of Pharmacy Practice, MMDU, Ambala Haryana - 133001, India.

${ }^{4}$ Department of Pharmacy, Anuradha College of Pharmacy, chikhali - 411062, India.

\begin{abstract}
A B S T R A C T
The current situation of Malignant Neoplasm is very different it's the fourth decade and despite much progress has been made in categorizing the environmental causes and cellular and molecular biological basis for this dreaded disease, Malignant Neoplasm commonly known as (Cancer) till now we did not understand the actual difference between the cancer cell and its normal counterpart. If we did not understand we cannot control, eliminate, treat cancer. The human genome sequence and its subsequent improvements in the sequence data are important steps to fully comprehend cancer cell biology. The current recent advances in the understanding of pharmacokinetic $\&$ pharmacodynamic behaviour of drug have offered a more rational approach to the development of optimal drug delivery system. The novel drug delivery systems (NDDS) are carriers which maintain the drug concentration in therapeutic range for a longer period of time. Nanotechnology, a new, novel focus of research evolved from the convergence and coalescence of many diverse scientific disciplines which includes Nanomedicine will have an impact on the key challenges in cancer therapy: nanotubes, Nanopores, and nanoparticles are the most promising applications for various Malignant Neoplasm treatments.
\end{abstract}

Key words: Nanotubes, Nano Particles, Nano Device, cancer cells, Novel Drug Delivery system, chemotherapy

A R T I C L E I N F O: Received 15 April 2019; Review Completed 05 July 2019; Accepted 03 August 2019; Available online 15 August2019

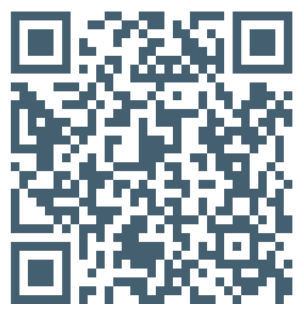

Cite this article as:

Mohammad khan Gayoor, Yezdani Umama, Kumar ayush, sadar mayur, Rav Shorabh, Sahu Lalit, Use of Nanoparticles for the Treatment of Malignant Neoplasm As a Cancer Targeted Drug Delivery System.Asian Journal of Pharmaceutical

Research and Development. 2019; 7(4):84-88, DOI: http://dx.doi.org/10.22270/ajprd.v7i4.513

*Address for Correspondence:

Mohammad Gayoor khan, Department of Pharmacy, Truba Institute of Pharmacy, Bhopal- 462038, India.

\section{INTRODUCTION}

A malignant Neoplasm is a group of diseases involving abnormal cell growth with the potential to invade or spread to other parts of the body. Such as tumours, which do not spread. Symptoms include a lump, abnormal bleeding, prolonged cough, unexplained weight loss, and a change in bowel movements. The nanotechnology; nanomedicine, nanoparticles are widely used for cancer cell treatment; they targeted particular site and show action it works within the therapeutic index.
Novel drug delivery systems are the new system ${ }^{1}$. Recent advances in the understanding of pharmacokinetic \& pharmacodynamic behaviour of the drug need an optimum approach for Drug Delivery system, drug concentration in therapeutic range for longer period of time ${ }^{2}$. There are several advantages of novel drug delivery systems over conventional drug delivery. This system is also using for treatment of various neurological diseases, Alzheimer's, Parkinson's, target cancer cells, etc ${ }^{3}$. 
There are several advantages of novel drug delivery systems over conventional drug delivery ${ }^{3}$.

- Frequent dosing and wastage of the drug may be reduced or excluded.

- Predetermined release rates of an extended period of time may be achieved.

- Better patient compliance may be ensured.

\section{Nanotechnology Relation}

A malignant Neoplasm is a group of diseases involving abnormal cell growth with the potential to invade or spread to Nanotechnology is the study of extremely small structures as it deals with materials in the size of 0.1 to 100 $\mathrm{nm}^{8}$. Nanotechnology works on the matter at dimensions in the nanometres scale length $(1-100 \mathrm{~nm})$ by a special scale that is being designed to calculate the activity of the nanoparticles (nanoscale) and hence can be used broadly in various fields ${ }^{4}$. Uses: Nanoparticles may also be used for the simultaneous tagging of multiple bio molecules ${ }^{6}$, both inside as well as outside the cells to monitor disease progression; Nanoparticles can be inserted into living cells as magnetic resonance contrast agents, cancer treatment, etc.

\section{Applications of Nanotechnology:}

1. Space exploration

2. Energy and Environment

3 . Early diagnosis and screening.

Nanoscale devices are 100 to 10,000 times smaller than human cells but are similar in size to large Biomolecules such as enzymes and receptors ${ }^{7}$. Nan scales that are smaller than or equal to $50 \mathrm{~nm}$ can easily pass all the materials and the one which are of $20 \mathrm{~nm}$ can move the diseased and other cells and are useful in the disease identification and can cross blood vessels. The materials which are larger like proteins and all they cannot move through this very easily and need a vehicle. Hence, targeted drug delivery vehicle is being used ${ }^{8}$. The difference between normal cells and tumour cells can be known by different approaches like topdown, bottom-up approach and this can be constructed by the use of nano devices. Coupled with the use of micro dissection techniques. These technologies helps us to identify the difference between the normal cells and the diseased cells this type of techniques are very much useful in the diagnosis of the tuberous cells for the diagnosis of cancer so that we can identify and target only the cells which are being diseased and put the drug into $i^{5}$. The major goal of this models and technologies are to eliminate the tuberous cells and not to cause harm to the normal cells so that the treatment should be accurate and the normal cells should not be affected.

\section{NANOTECHNOLOGY AND TARGETED DRUG DELIVERY}

The drug delivery is the major problem in cancer as we need to target a particular tissue and cells and this can be effectively done by using nanotechnology. If nanotechnology is used in an appropriate way then it will help us in proper dosing and drug delivery of almost all the drugs and can increase effectiveness of the drugs ${ }^{4}$. Drug carriers like nanosized liposomes' can be used for the stability and efficacious supply of the compounds in the blood so that it can enter into the circulation and can easily cross the BBB to attack on the tumor cells. The best way to increase the efficacy and reduce the toxicity of a cancer drug is to direct the drug to its target and maintain its concentration at the site for a sufficient time for therapeutic action to take effect example - lipid cationic nanoparticles coupled to an integrin-targeting ligand were shown to deliver genes selectively to angiogenic blood vessels in tumor-bearing mice. As the therapeutic part of the noncomplex, a mutant RAF gene was coupled to the particle for transfection and expression in the tumor cells; expression of this mutant gene was shown to block angiogenesis in this model $^{6}$. By using these techniques bioavailability of drugs, target detection, targeted drug delivery can be increased by the use of nanotechnology structure mediated procedure. Nanoscale drug delivery systems can be implemented within pulmonary therapies, as gene delivery vectors, and in stabilization of drug molecules that would otherwise degrade too rapidly. Additional benefits of using targeted Nano scale drug carriers are reduced drug toxicity and more efficient drug distribution ${ }^{9}$. The efficacy enhances the gene therapy. This protocol was shown to improve effectiveness of many prostatic and liver cancer cells and to increase the targeted drug delivery. Monoclonal antibodies are good targeting vehicles for nanoparticles with greater degree of success. In in vivo culture aptamers are generated by using evolution methods, and in vivo culture antigens are targeted by molecules that are with higher affinity ${ }^{9}$. Prostate cancer cells can be treated by the use of antigens called PEGcoated nanoparticles.

There are numerous examples of similar type targeting of nanoparticles and this area of research promises to provide important weapons in the arsenal for developing a cure for cancer. One of the ultimate goals of nanotechnology is to create medically useful Nano devices that can function inside the body ${ }^{2}$. It is envisioned that Nano devices will be hybrids of biologic molecules and synthetic polymers that can enter cells and the organelles to interact directly with DNA and proteins additionally; nanomedicine also has an effective and positive impact on the various types of cancer treatment and can overcome various types of challenges that are faced during the cancer treatment. This can be effectively done by the use of nano tubes, nano shells and other materials that are known to cure cancer. The gold Nano shell-antibody complex can be used to ablate breast cancer cells ${ }^{5}$. Nano shells have a core of silica and a metal outer layer they have an advanced permeability in the cancer cells and are very effective in that.

\section{Specific Receptor Targeting}

In many anticancer therapies it was found that folic acid is one of the most important components that are known to be causing over expression. Utilizing this concept, researchers are designing the surface of nanoparticles with folic acid as a targeting agent for the delivery of pHPMA conjugated daunomycin in four murine tumor models ${ }^{10}$. Folic acid targeted daunomycin-HPMA conjugates were found to 
increase the no of survivors and cure of the cells in high number of cells.

\section{Nanotechnology in Cancer Targeting}

Use of nanotechnology is a very many important concept of the treatment and the use of accurate dosage of the drug this can be done by making various kinds of modifications in the formulation of the drug by changing their size, shape, chemical properties and by other modifications and this can be helpful in the targeting of the cells in the form of passive targeting.

\section{Active Targeting}

In case of active targeting, nanoparticles containing the chemotherapeutic agents are designed in such a way as they directly interact with the defected cells ${ }^{11}$. Active targeting is based on molecular recognition. Targeted delivery system has three main components: (i) an apoptosis-inducing agent (anticancer drug), (ii) a targeting moiety-penetration enhancer, and (iii) a carrier. A variety of substances are used to construct a nanoparticle ${ }^{7}$.

\section{POLYMER MATERIALS}

An accurate review of biodegradable polymeric materials that show tremendous response for drug delivery applications is being compiled ${ }^{11}$. Biodegradable polymer nanoparticles, typically consisting of polylactic acid (PLA), polyglycolic acid (PGA), or a copolymer of PLA and PGA, are being investigated for the delivery of proteins and genes, vaccines and anticancer drugs Dendrimers, a unique class of polymers, are highly branched macromolecules whose size and shape can be precisely controlled ${ }^{6}$. Dendrimers are shown in figure 1.1 The well-defined structure, monodispersity of size, surface functionalization capability, and stability are properties of dendrimers that make them attractive drug carrier candidates ${ }^{13}$.

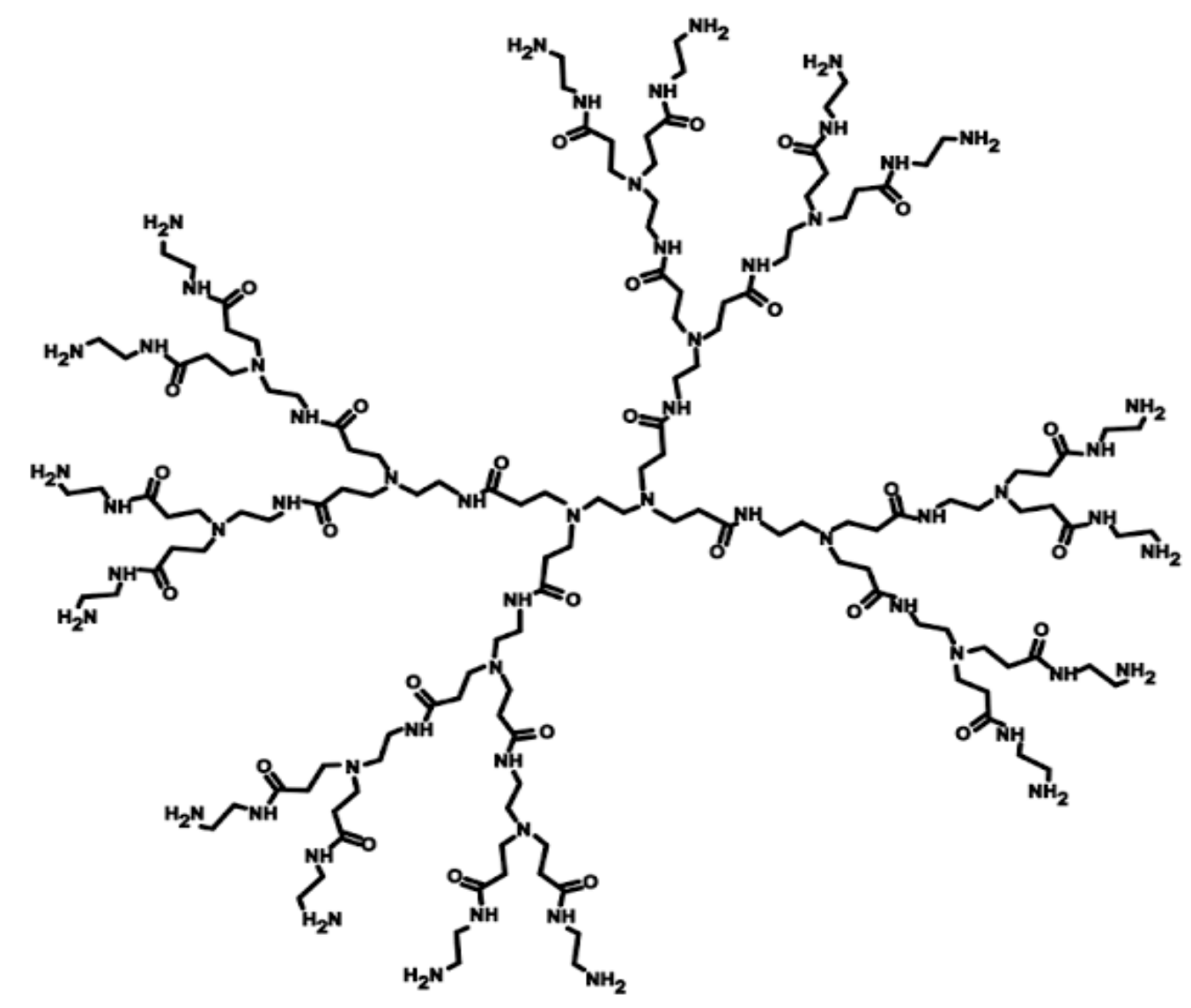

Figure 1.1: Example chemical structure of Chemical structure of a polyamidoamine dendrimer

\section{METAL STRUCTURES}

The hollow type metal Nano shells are being investigated for drug delivery applications different types of methods are being used for this and one major type of it is by using typical metals where these are wrapped around the material. Typical metals include gold, silver, platinum, and palladium $^{7}$. Nanoparticles can be used as thermal release triggers when irradiated with infrared light or excited by an alternating magnetic field bimolecular conjugation methods of metals include bifunctional linkages, nano bead interactions ${ }^{14}$.

\section{NANOMEDICINE AND NEW DRUG THERAPIES FOR CANCER}

All the drugs are either from the natural or synthetic products and are being prepared they have different essence 
and effect based on their use and effect ${ }^{13}$. The most widely used drug is aspirin which is even highly effective and its length is only $0.6 \mathrm{~nm}$ on the other hand the drugs in monoclonal antibiotics which are not much effective are $30 \mathrm{~nm}$ in length hand that of protein is $5 \mathrm{~nm}$ diameter and natural proteins of similar size may be used as a therapeutic ${ }^{2}$. Double-stranded DNA in the nucleus is about $2.5 \mathrm{~nm}$ in width, but totals 2 meters in length in the mammalian cell, an astounding example of "Nano packing" in a 2- to 5- $\mu \mathrm{m}$-diameter nucleus ${ }^{15}$. Chemist around the world both from research field and medical field are being changing and manipulating the structure of the drug by using nanometres for a better future in medicine. The difference is that they will now be joined by a wide variety of scientists from a number of disciplines normally not involved in drug research.

\section{Limitations of Conventional Chemotherapy}

The dividing cancerous cells are being destroyed by the use of Conventional chemotherapeutic agents. This is why chemotherapy also damages normal healthy cells that divide rapidly such as cells in the bone marrow, macrophages, digestive tract, and hair follicles the main drawback conventional chemotherapy is they are not able to give an accurate action and effect only on the particular cancerous cells $^{16}$. Because of this chemotherapeutic agents causes many number of side effects some of them are so deadly that completely kills the normal cells.

\section{WHAT ARE THE BASICS REQUIREMENTS FOR AN EFFECTIVE AND SAFE CANCER DRUG?}

The drug concentration is the blood is very important for the drug to reach the target cells and get absorbed and work properly in a way that all of the cells should get the proper amount of drug ${ }^{4}$.

The drug must have high differential toxicity toward the tumor or a favourable therapeutic window. All these points are being noted and mentioned while doing clinical research on cancer cells and there medicine it is mainly carried out in the monoclonal antibiotic which is probably the new best treatment of cancer ${ }^{10}$. They are being used as imaging vehicles, for drug targeting, as drug carriers, and as the drug itself. There are 9 or more FDA-approved antibodies approved for clinical use in cancer, and many more are being evaluated in clinical trials. The mechanism of action of the antibodies includes receptor ligand binding competition; interference with receptor function; antibodydependent, cell-mediated cytotoxicity; complementdependent cellular cytotoxicity; or, perhaps, a combination of the above. Even more effective effect can be expected to be produced when they are combined with the toxin a complete study can be done ${ }^{5}$. Whereas Development of many drugs will be the result of the application of nanotechnologies that have been in place for many years. For example, nuclear magnetic resonance and $\mathrm{x}$-ray crystal structures of target proteins and their ligands or substrates are being used as the template for the rational design of new drugs $^{3}$. The target may be enzymes or receptor-ligand proteins. Inhibitors of enzymatic activity are, in general, easier to design than blockers of protein-protein interactions ${ }^{12}$. As an example, the first successful drug approved for the treatment of chronic myeloid leukemia (CML), Gleevec, is an inhibitor of the tyrosine kinase mutant, BCR-ABL. In most of the patients the drugs are being administered is completely dependent on the drug flow and absorption ${ }^{17}$. Where the Gleevec-resistant leukemia often returns through the mutations in the active site. By doing careful examination of this we can diagnose the effect of it compared to the normal drug and it was found that this is even more effective than the normal drug. Chronic myeloid leukemia can be cured by the use of this method and is very much effective. Drugs derived from nucleic acids are now making an impact on the nanomedicine scene ${ }^{5}$. Antisense technology exploits the use of oligonucleotides in the range of 15 to 20 nucleotides to block the function of an RNA target. This process did not succeed in the initial stages and cure for prostate, breast, pancreatic, lung, colorectal, melanoma, and brain cancers. This is the best example for nanotechnologies can be used to increase the effectiveness of existing drugs, facilitating the use of lower dosages to decrease toxicity. Cancer immunotherapy may provide a relatively benign, no chemotherapeutic method of destroying tumors. Nanoshells, in this case, is composed of a silica core surrounded by a thin gold metal shell and will absorb energy (heat up) when exposed to the appropriate wavelength of light ${ }^{7}$. When these Nanoshells were injected into mice and the nanoparticles were allowed to accumulate in implanted tumors. In this the drugs effect only the tissues which are diseased were affected and did not cause any effect on the other cells. The tumors are then illuminated with a near-infrared diode laser to heat the tumor and cause cellular destruction. By this protocol, all the tumors were ablated, and the mice remained tumor-free for many months ${ }^{17}$.

\section{RESULT}

The Nanotechnology is used for cancer research \& treatment is recognized by Targeted drug delivery system via nano medicine they target particular site and show particular actions various type of cancer should be treated by using this discipline few FDA approved drugs are also used for brain cancer, colon cancer etc. In appropriate settings, thermal ablation methods could be used to replace surgical resection of tumors, and targeted therapies and immunotherapy as a substitute for toxic chemotherapy hence Nano medicinal methods being developed have a good chance of achieving this goal with much less damage to normal tissue than existing therapeutic index.

\section{CONCLUSION}

The cancer therapy is being increased and developed by the use of nanotechnology. Cancer has also being evolved as much as that of the heart tissues and it has also evolved in the coming world as much as that of any other disease. Cancer has been one of the leading causes of death in today's world. Although cancer may be more complex than cardiovascular disease, it is not inconceivable that lifestyle changes (smoking cessation) and new drugs developed from Nano technological and other medical advances could treat this very effectively. This will allow the creation of nontoxic, targeted small-molecule drugs for use in the 
oncology clinic. Due to the complexity of cancers, a combination of approaches will likely be needed for the effective elimination of all tumor cells. As cancer is one of the major cause of death and even a disease which is spreading very vastly hence its treatment and a proper cure for it is to be known and now scientist are able to cure it very effectively in a proper duration of time. More amount of these technologies applied can very positively this.

\section{ACKNOWLEDGEMENT}

First of all I would like to Thank my Co- Author Ms. Umama Yezdani (Department of clinical and Pharmacy Practice) MRM college of Pharmacy Hyderabad, India. The authors are thankful to Dr. Rajesh Singh PAWAR, PhD. Principal Truba Institute of Pharmacy for ceaseless encouragement during the study.

\section{CONFLICT OF INTERESTS}

The authors declare that there is no conflict of interests regarding the publication of this paper.

\section{REFERENCES}

1. Gayoor Khan, Umama Yezdani, Rohit Verma, Raqshan Jabeen,Pradeep Sintha.Detection of Phlebovirus by using qualitative Real time (RT) PCR and application of silver nanoparticles to control it. World J Pharm Pharm Sci.2018; 7(11):936-52.

2. Mohd.Gayoor Khan.The Novel Drug Delivery System. World J Pharm Pharm Sci.2017; 6(7):477-487.

3. Unama Yezdani, Mohd. Gayoor Khan, Fazal Khan, Arvind Verma, Nilesh Kushwah, Rohit Verma.The Drug Targeting in Alzheimer's or Applications \& it's Hazards. World J Pharm Pharm Sci.2017; 7(11):1532-1549.

4. H.S Chandel., Sharad P.Panday, Arvind Dangi, Ashish chaurasia, Mohd.Gayoor khan et al. Development of Targeted Drug delivery. International Journal of research methodology Ijrm.Human. 2017; $1(2): 30-34$.
5. Kushwah Nilesh,Yezdani Umama,Mohammad Gayoor khan,Manish kushwah, Kumar Ayush.The Fundamental of Novel Drug Delivery System; Methodology, Role of Nanotechnology; Nanoparticles in Pharmaceutical Research. International Journal of Emerging Technologies and Innovative Research 2019; 6(6):140-146.

6. Mohd. Gayoor Khan, Nilesh Kushwaha, Fazal Khan, Vipul Patel. Microencapsulation. International Journal of research methodology. 2017; 1(2):35-42.

7. Umama Yezdani, Mohd. Gayoor Khan, Nilesh Kushwah, Arvind Verma,Fazal Khan. Application of Nanotechnology in Diagnosis and treatment of various disease and it's future advances in medicine. World J Pharm Pharm Sci.2018; 7(11):1611-1633.

8. Yezdani Umama,Venkatajah G, Rav Shourabh, Roshan Kumar, Arvind Verma, Ayush Kumar, Md. Khan Gayoor et al. Topic-The scenario of pharmaceuticals and development of microwave as;sisted extraction technique. World J Pharm Pharm Sci.2019; 8(7):1260-1271.

9. H. Zhou and C. Liu. Microwave assisted extraction of solanesol from tobacco leaves.J. Chromatograph. A. 2006; 1129:135-39.

10. L. Wang and C.L. Weller. Recent advances in extraction of nutraceuticals from plants. Trends Food Sci. Technol.2006; 17:300-12.

11. S. Karthikeyan, R. Bal Subramanian and S.W. See. Optimization and validation of a low temperature microwave assisted extraction method for analysis of polycyclic aromatic hydrocarbons in airborne particulate matter. Talanta, 2006; 69: 79- 86.

12. M. letellier, H. Budzinski, L. Charrier, S. Capes and A.M. Dorthe. Optimization by factorial design of focused microwave assisted extraction of polycyclic aromatic hydrocarbons from marine sediment. J. Anal. Chem. 1999; 364:228-37.

13. C.W. Huie. A review of modern sample preparation techniques for the extraction and analysis of medicinal plants. Anal. Bioanal. Chem, 2002; 373: 23-30.

14. Drug delivery systems third edition Vasant V. Ranade, John B. Cannon, Pg no. 56-63.

15. Gobin AM, O'Neal DP, Watkins DM, Halas NJ, Drezek RA, near infrared laser-tissue welding using Nano shells as an exogenous absorber. Lasers Surg Med, 2005; 37: 123-129.

16. Nishizawa M, Menon VP, Martin Cr: Metal Nano tubule membranes with electrochemically switchable ion-transport selectivity. Science, 1995; 268: 700-702.

17. Targeted delivery of small \& macromolecular drugs. Editor- Ajit S. Narang; Page no. 78-83. 